\title{
Nanobiomedicina de Nanopartículas Magnéticas de Óxido de Ferro: Considerações e Perspectivas
}

\author{
Joyce F. L. Sousa \& Luciana R. Guilherme
}

Com o advento da nanotecnologia farmacêutica, novas formulações, sistemas de liberação de fármacos e métodos de diagnósticos foram disseminados nos últimos anos. Nanopartículas magnéticas de óxido de ferro (SPIONs) surgem como uma perspectiva na identificação precoce de tumores, por meio da ressonância magnética nuclear. Como carreadores de fármacos demonstram reduzir a toxicidade e direcionar o fármaco ao síto de ação desejado, além de demostrarem eficiência no tratamento de tumores, por provocarem hipertemia na região específica da lesão. O objetivo deste trabalho é apresentar algumas das aplicações biomédicas de nanopartículas magnéticas de óxido de ferro.

Palavras-chave: Nanopartículas magnéticas de óxido de ferro; aplicação biomédica; liberação de fármaco.

With the advent of nanotechnology pharmaceutical new formulations, drug delivery systems and diagnostics methods have been widespread in recent years. Magnetic iron oxide nanoparticles (SPIONs) appear as a prospect in the tumor early identification by nuclear magnetic resonance. As carriers of drugs shown to reduce toxicity and direct the drug to the sito desired action. Besides demonstrate efficiency in tumor treatment by hyperthermia cause the specific lesion region. The objective of this paper is to present some of the biomedical applications of magnetic nanoparticles of iron oxide.

Keywords: Iron oxide nanoparticles magnetics; magnetic hyperthermia; drug delivery. 


\section{Introdução}

A nanotecnologia é uma ciência multidisciplinar promissora, a qual possui diversos campos de atuação e está associada à manipulação da matéria em escala nanométrica, ou seja, uma escala tão pequena quanto à de um bilionésimo do metro. Um nanômetro (nm) possui dimensões entre 1-100 $\mathrm{nm}^{1}$. Produtos obtidos a partir da nanotecnologia podem apresentar características interessantes como: tolerância à temperatura, cores, reatividade química, condutividade elétrica ou mesmo exibir força de intensidade extraordinária ${ }^{2}$. Essa tecnologia já está dispersa em diversas áreas, como tecnologia para os eletrônicos, física, agricultura, química, farmácia e outros ${ }^{3}$.

Nanomedicina é a ciência envolvida no desenvolvimento, caracterização, sistemas de liberação e direcionamento de fármacos, diagnóstico, tratamento de doenças e outras aplicações ${ }^{2}$.

O setor farmacêutico é uma das áreas que mais vem investindo no desenvolvimento de pesquisas, com o objetivo de melhorar as propriedades físico-químicas de alguns fármacos que prejudicam a estabilidade, biodisponibilidade, a eficácia, o transporte e a liberação, trazendo, assim, melhor conforto posológico, reduzindo toxicidade e feitos colaterais ao paciente ${ }^{3,4}$.

Uma abordagem promissora para o desenvolvimento de sistemas de liberação controlada de fármacos é baseada na associação de nanopartículas com materiais magnéticos, fluidos magnéticos, que são suspensões coloidais estáveis, contendo nanopartículas magnéticas de óxido de ferro dispersos em veículos líquidos orgânicos ou inorgânicos ${ }^{4}$.

Nanopartículas magnéticas de óxido de ferro são materiais que têm sido amplamente utilizados em medicina para direcionamento do fármaco, sistemas de liberação controlada, diagnósticos via imagiologia e aplicações terapêuticas ${ }^{5}$. Já foram realizados vários estudos demonstrando que partículas nanomagnéticas aumentam a eficiência e a qualidade de diagnósticos realizados por ressonância magnética ${ }^{6}$.

Um objetivo importante na terapia de liberação controlada é o de melhorar a disponibilidade do fármaco no seu local de ação, minimizando sua exposição a sítios não alvos. Este sistema permite que o material alcance, por meio da aplicação de campo magnético externo, o local desejado e atinja a concentração terapêutica do fármaco ${ }^{6}$.

Outro estudo relacionado a nanopartículas magnéticas de óxido de ferro é seu emprego frente a campos magnéticos alternados que podem induzir à hipertemia. A hipertermia pode ser aplicada para o tratamento de câncer de cabeça e pescoço. Análises patológicas demonstraram a diminuição de células epiteliais do tumor associado com o tratamento de hipertemia ${ }^{7}$. Novas pesquisas indicam que essa tecnologia também pode ser útil na retirada de metais pesados no tratamento de água, em biomateriais, biofilmes $^{8}$, tratamento para cardiopatia ${ }^{9}$ e outros.

Arelevância do tema é motivada pelo desenvolvimento crescente da nanotecnologia. O objetivo deste estudo é mostrar os principais estudos de aplicações biomédicas de nanopartículas magnéticas de óxido de ferro e suas perspectivas de uso.

\section{NANOPARTÍCULAS MAGNÉTICAS}

As nanopartículas magnéticas de óxido de ferro (SPIONs) do presente estudo são formadas por óxidos de ferro de maguemita $\left(\gamma-\mathrm{Fe}_{2} \mathrm{O}_{3}\right)$ e de magnetita $\left(\mathrm{Fe}_{3} \mathrm{O}_{4}\right)$ por síntese de criopreciptação ${ }^{10}$. AApós a síntese, pode-se preparar o fluido magnético ou ferrofluido, um coloide. Este, por sua vez, é constituído pela suspensão de partículas magnéticas de óxido de ferro em escala nanométrica. Por tratar-se de escala nanométrica, as nanopartículas apresentam uma grande área superficial e podem apresentar agregação de suas partículas. Por esse motivo, faz-se necessário minimizar as aglomerações por meio do processo de estabilização do fluido, realizando o revestimento, a cobertura ou a funcionalização das partículas ${ }^{1}$. Nesse processo, as nanopartículas magnéticas de óxido de ferro são revestidas em sua superfície com tensoativos, surfactantes ou polímeros biocompatíveis, de modo a manter a estabilidade coloidal do fluido ${ }^{11}$. Os surfactantes favorecem uma repulsão eletrostática no fluido e, desse modo, contribuem para a não aglomeração das partículas. Os surfactantes são responsáveis também por manter propriedades coloidais em $\mathrm{pH}$ próximo de 7 , em altas temperaturas e quando submetidos a campos magnéticos alternados ${ }^{12}$. 
O grande interesse em aplicações biomédicas é devido às suas propriedades superamagnéticas que consistem em apresentar magnetização apenas na presença de um campo magnético externo. O suparamagnetismo apresenta um momento magnético atômico, presença de átomo com elétron desemparelhado que, sob influência de campo magnético externo, magnetiza-se e se orienta em direção a este campo. Entretanto, na ausência do campo magnético externo não apresenta magnetização ${ }^{13}$.

Estudos vêm demonstrando que quando se utiliza a associação das propriedades Superparamagnéticas e a funcionalização da sua superfície com surfactantes, as nanopartículas magnéticas podem ser utilizadas principalmente em aplicações biomédicas, como a vetorização com biomarcadores ${ }^{14}$, liberação de fármacos conjugados $^{13}$, diagnóstico por imagem por ressonância magnética nuclear, hipertemia magnética ${ }^{15}$, etc.

Como diagnóstico, as nanopartículas podem ser empregadas como contrasteno diagnóstico por imagem por ressonância magnética nuclear para aumentar a qualidade de imagens e na localização de neoplasmas precoces ${ }^{7}$. Como terapia, pode-se citar a hipertemia magnética e o direcionamento localizado de quimioterápicos para o tratamento de tumores ${ }^{8}$. Além disso, por meio de marcadores e surfactantes, as nanopartículas possibilitam incorporação e direcionamento de fármacos acoplados a sua superfície, promovem a liberação controlada e direcionada no sítio-alvo desde que guiadas por gradientes de campos magnéticos externo ${ }^{7}$.

\section{DIAGNÓSTICO POR RESSONÂNCIA MAGNÉTICA NUCLEAR}

Para que um tratamento terapêutico seja eficaz, é necessário que o paciente receba um diagnóstico precoce e preciso ${ }^{5}$. A ressonância magnética nuclear é um procedimento não invasivo que possui alto poder de resolução na qualidade das imagens, sendo possível visualizar aspectos fisiológicos e anatômicos simultaneamente. Ela é muito utilizada para diagnósticos, exames pré-cirúrgicos e monitoramento de terapias e, também, é capaz de gerar imagens $3 \mathrm{D}^{16}$.

O mecanismo da ressonância magnética nuclear consiste no alinhamento de prótons de hidrogênio a um campo magnético externo. Os prótons de hidrogênio da água presente no tecido a ser diagnosticado, quando submetido a uma frequência de radiação, sofre uma perturbação e quando esses prótons voltam ao seu estado normal, a imagem é obtida. Tecidos diferentes apresentam velocidades diferentes de retornos dos prótons. E devido a esta peculiaridade, as imagens apresentam contrates de contornos para diferenciar as estruturas biológicas de interesse $\mathrm{e}^{17}$.

Como a atividade metabólica de células doentes é diferente de células sadias, a ressonância magnética promove a grande vantagem de identificar essas diferenças, por meio das imagens. Dessa forma, é possível monitorar o avanço das doenças, sendo uma poderosa ferramenta para o diagnóstico e tratamento neoplásico ${ }^{18}$. Para aprimorar o diagnóstico, contrates com nanopartículas suparamagnéticas de óxido de ferro foram desenvolvidos com o objetivo de: melhorar o contraste das imagens, modular o tempo de permanência do contraste no organismo e monitorar células específicas ${ }^{16}$.

Muitas formulações de nanopartículas magnéticas foram desenvolvidas e já estão no mercado como contraste em ressonância magnética nuclear (Endorem, Feridex IV ${ }$, Lumiren ${ }^{\circledR}$ e Combidex $\left.{ }^{\circledR}\right)$. Pesquisas atuais na área de ressonância magnética nuclear para a terapia oncologia buscam aperfeiçoar as interações das nanopartículas, a fim de melhorar a nitidez das imagens, reduzir a quantidade administrada de contrate e aprimorar a funcionalização das nanopartículas para que o contrate atinja o sítio desejado ${ }^{19}$.

Estudos com marcadores de peptídeos de câncer de próstata associados às nanopartículas magnéticas de óxidos de ferro observaram acúmulo de material em região específica, identificando com precisão o local da lesão. Além disso, apresentaram melhor qualidade nas imagens por ressonância magnética nuclear ${ }^{17}$. Marcadores fluorescentes também estão sendo utilizados em testes combinados com nanopartículas para entrega de drogas guiadas por imagem como uma nova plataforma de teradiagnóstico ${ }^{16}$.

Outro estudo verificou que a associação de nanopartículas magnéticas a anticorpos monoclonais permitiu a detecção precoce de micrometástase em tumores de cérebro ${ }^{20}$. Além de agentes de contraste para diagnóstico, estudos destacam o uso desses sistemas magnéticos para liberação controlada de fármacos ${ }^{19}$. 


\section{Sistema de Vetorização e Liberação de Fármacos}

Um dos grandes problemas na administração de medicamentos refere-se ao grande desconforto do paciente em administrar várias doses para manter o nível sérico do fármaco no organismo, a grande quantidade de efeitos colaterais e os efeitos tóxicos devido a não seletividade dos fármacos ${ }^{15}$.

Com o intuito de melhorar esses problemas, as nanopartículas, quando associadas a outros fármacos, podem propiciar sinergismo e aumentar a eficácia frente ao tratamento de algumas patologias, devido principalmente a sua capacidade de vetorização e liberação controlada de fármacos ${ }^{30}$.

O transporte e liberação controlada de fármacos pelas nanopartículas magnéticas de óxidos de ferro são possíveis devido as suas propriedades magnéticas de serem orientadas por campo magnético e também pelas suas propriedades adquiridas após sua funcionalização por polímeros, tensoativos ou surfactantes biocompatíveis. Esses agentes de funcionalização possuem a capacidade de modular a liberação do fármaco no organismo ${ }^{11}$.

Propondo-se potencializar fármacos com conhecidos efeitos terapêuticos, as nanopartículas magnéticas surgem como uma promissora classe de nanocarreadores com o objetivo de carrear o fármaco pela circulação sanguínea até o sitio-alvo ${ }^{22}$.

A possibilidade de carrear o fármaco até o sítio-alvo, é muito interessante, uma vez que, direcionando o fármaco até seu local de ação, pode-se evitar a interação deste a outros órgãos ou tecidos que não sejam de interesse de atuação do fármaco. Dessa maneira, podem-se minimizar os efeitos colaterais e tóxicos ${ }^{6}$. Com a vetorização, sugere-se também que o controle de liberação do fármaco no sítio-alvo possa aumentar o intervalo entre as doses para proporcionar maior conforto ao paciente e consequentemente maior adesão ao tratamento ${ }^{20 .}$

\section{Hipertemia}

Hipertermia é o aumento acima do normal da temperatura corpórea. Altas elevações de temperatura podem alterar a homeostasia corporal e causar danos em tecidos. A partir desse conceito, vários estudos são realizados com o objetivo de provocar o aumento da temperatura em lugares específicos, bem como de provocar danos seletivamente em regiões neoplásicas. Isso causa a morte das células cancerosas, uma vez que essas são menos resistentes a elevações de temperaturas que células normais e saudáveis ${ }^{3,22}$.

Para provocar aumento da temperatura em região localizada, as nanopartículas magnéticas de óxido de ferro surgem como promissoras para o tratamento terapêutico contra o câncer, sendo denominado como hipertemia magnética ${ }^{18}$. Devido às suas propriedades de superparamagnetismo, as nanopartículas de óxido de ferro absorvem energia e liberam calor na presença de um campo alternado magnético externo ${ }^{22}$. É necessário utilizar os procedimentos de ressonância magnética nuclear e de vetorização, para carrear as nanopartículas ao sítio-alvo e provocar a hipertemia magnética ${ }^{23}$.

Para promover o processo de hipertemia, o fluido de nanopartículas superparamagnéticas de óxido ferro devem ser estabilizados e funcionalizados para se associarem a anticorpos e serem usadas como vetores até o sítio-alvo pretendido na região do tumor ${ }^{13}$. As nanopartículas são orientadas por meio de um campo magnético externo até o local do tumor. Com a aplicação de correntes alternadas de força e frequência, as nanopartículas absorvidas pelas células cancerosas dissipam calor e provocam a elevação da temperatura, em torno de $41^{\circ} \mathrm{C}$ a $46{ }^{\circ} \mathrm{C}$, no local do tumor e provocam consequente lise das células tumorais neoplásicas ${ }^{24}$.

\section{Considerações Finais}

Nanopartículas magnéticas de óxido de ferro possuem propriedades superamagnéticas que conferem a essas a capacidade de serem orientadas a um campo magnético externo. Estudos de sistemas de liberação de fármacos, com nanopartículas magnéticas, mostram-se promissores para direcionamento do fármaco ao seu local de ação esperado, proporcionando melhor eficiência ao tratamento ao paciente. Imagens por ressonância magnética nuclear utilizando contrastes, a partir de nanopartículas magnéticas de óxido de ferro, mostraram melhor nitidez nas imagen ${ }^{4}$. Além disso, estudos relatam que se essas nanopartículas forem funcionalizadas com marcadores específicos, essas podem ser utilizadas para o diagnóstico mais preciso de patologias e serem úteis 
na identificação precoce do câncer. Estudos demonstram também que outra promissora terapia no combate ao câncer é a hipertemia magnética.

Espera-se que outros estudos possam corroborar com mais informações a cerca das aplicações biomédicas de nanopartículas magnéticas de óxido de ferro.

\section{Referências Bibliográficas}

1. Hussein-Al-Ali, S. H.; El Zowalaty, M. E.; Kura, A. U.; Geilich, B.; Fakurazi, S.; Webster, T. J.; Hussein, M. Z. Biomed Res. Int. 2014, 2014, 651831 .

2. Kolhatkar, A. G.; Jamison, A. C.; Litvinov, D.; Willson, R. C.; Lee, T. R. Int. J. Mol. Sci. 2013, 14, 15977.

3. Marszałł, M. P. Pharm. Res. 2011, 28, 480.

4. Babincova, M.; Babinec, P. Biomed Pap Med Fac Univ Palacky Olomouc Czech Repub. 2009, 153, 243.

5. Xiao, Y.; Lin, Z. T.; Chen, Y.; Le, D. E.; Bin, J. Int. J. Nanomedicine 2015, 10, 1155.

6. Linemann, T.; Thomsen, L. B.; du Jardin, K. G.; Laursen, J. C.; Jensen, J. B.; Lichota, J.; Moos, T. Pharmaceutics 2013, 5, 246.

7. Enochs, W. Scott; Harsh, Griffith; Hochberg, Fred; WeisslederRalph J. Magn. Reson. Im. 1999,9, 228.

8. Trever Todd, Zipeng Zhen, Wei Tang, Hongmin Chen, Geoffrey Wang, Y.-J.; Chuangc, Kayley Deaton, Zhengwei Panc, and J. X. Nanoscale 2012, 29, 997.

9. Hussein-Al-Ali, S. H.; El Zowalaty, M. E.; Hussein, M. Z.; Ismail, M.; Webster, T. J. Int. J. Nanomedicine 2014, 9, 549.

10. Xiong, F.; Wang, H.; Feng, Y.; Li, Y.; Hua, X.; Pang, X.; Zhang, S.; Song, L.; Zhang, Y.; Gu, N. Sci. Rep. 2015, 5, 8579.

11. Liakos, I.; Grumezescu, A. M.; Holban, A. M. Molecules 2014, 19,12710 .

12. Mody, V. V.; Cox, A.; Shah, S.; Singh, A.; Bevins, W.; Parihar, H. Appl. Nanosci. 2013, 4, 385.

13. Dorniani, D.; Hussein, M. Z. Bin; Kura, A. U.; Fakurazi, S.; Shaari, A. H.; Ahmad, Z. Int. J. Mol. Sci. 2013, 14, 23639.

14. Wahajuddin; Arora, S. Int. J. Nanomedicine 2012, 7, 3445.

15. Jayant, R.; Atluri, V.; Agudelo, M.; Sagar, V.; Kaushik, A.; Nair, M. Int. J. Nanomedicine 2015, 10, 1077.

16. Mihaela Istrate, C.; Holban, A. M.; Grumezescu, A. M.; Mogoantă, L.; Mogoşanu, G. D.; Savopol, T.; Moisescu, M.; Iordache, M.; Vasile, B. Ş.; Kovacs, E. Rom J Morphol Embryol 2014, 55, 849 .

17. Busquets, M. A.; Sabaté, R.; Estelrich, J. Nanoscale Res. Lett. 2014, 9 ,
18. Singh, D.; McMillan, J. M.; Kabanov, A. V; Sokolsky-Papkov, M.; Gendelman, H. E. Nanomedicine (Lond). 2014, 9, 501.

19. Hussein-Al-Ali, S. H.; El Zowalaty, M. E.; Hussein, M. Z.; Geilich, B. M.; Webster, T. J. Int. J. Nanomedicine 2014, 9, 3801.

20. Berman, S. C.; Walczak, P.; Jeff W.M. Wiley Interdiscip. 2011, 3, 343.

21. Wankhede, M.; Bouras, A.; Kaluzova, M.; Hadjipanayis, C. G. Expert Rev. Clin. Pharmacol. 2012, 5, 173.

22. Ang, L.; Chen, B.; Liu, S.; Wang, R.; Hu, S.; Xia, G.; Tian, Y.; Cai, X. Int. J. Nanomedicine 2012, 7, 4109.

23. Lee, J. H.; Kim, J. W.; Cheon, J. Mol. Cells 2013, 35, 274.

24. Huang, G.; Chen, H.; Dong, Y.; Luo, X.; Yu, H.; Moore, Z.; Bey, E. a.; Boothman, D. a.; Gao, J. Theranostics 2013, 3, 116.

25. Sensenig, R.; Sapir, Y.; MacDonald, C.; Cohen, S.; Polyak, B. Nanomedicine (Lond). 2012, 7, 125.

\section{Joyce F. L. Sousa* \& Luciana R. Guilherme}

Ciências Exatas e Tecnológicas. Universidade Estadual de Goiás. Caixa Postal 459, CEP 75001-970, Anápolis, GO.

*E-mail: joycelouzaa@gmail.com 